\title{
Value-Driven Design - A methodology to Link Expectations to Technical Requirements in the Extended Enterprise
}

\author{
Ola Isaksson ${ }^{1}$, Mario Kossmann², Marco Bertoni3,4, Hakki Eres ${ }^{5}$, Anne Monceaux ${ }^{6}$, Alessandro Bertoni ${ }^{4}$, \\ Steve Wiseall ${ }^{7}$ Xinwei Zhang ${ }^{8}$ \\ ${ }^{1}$ GKN Aerospace Engine Systems Sweden (Ola.Isaksson@gknaerospace.com) \\ 2AIRBUS (Mario.Kossmann@airbus.com) \\ 33Blekinge Institute of Technology (marco.bertoni@bth.se) \\ ${ }^{4}$ Luleå University of Technology (marco.bertoni@ltu.se, alessandro.bertoni@ltu.se) \\ 5University of Southampton (Hakki.Eres@soton.ac.uk) \\ 6EADS Innovation Works (Anne.Monceaux@eads.net) \\ ${ }^{7}$ Rolls-Royce plc (Steve.Wiseall@rolls-royce.com) \\ 8Université de Toulouse (xwzhang@etud.insa-toulouse.fr)
}

Copyright $(\subset 2013$ by the authors. Published and used by INCOSE with permission.

\begin{abstract}
Current systems engineering (SE) standards do not address 'Value' in much detail. Yet, understanding what drives the generation of stakeholder value in a given business context, is fundamental to promoting a common and clear vision throughout the extended enterprise, of what should be the focus of their early, conceptual work at all levels of development. This paper presents a Value-Driven Design (VDD) methodology designed to strengthen the value and requirements maturation process within an extended enterprise setting. The work presented is the result of a three and a half year European program (CRESCENDO) within the aerospace sector. The VDD methodology is introduced and explained in an industrial aircraft development context and a selection of enabling methods and tools associated to the VDD methodology is presented.
\end{abstract}

\section{Introduction and Background}

Traditionally, the basis for any work at any level of this extended enterprise would be the technical requirements, which are signed off as part of the respective contracts between directly interfacing partners within the extended enterprise. However, there are strong pressures to develop ever faster, better and cheaper. In order to respect the short development schedules within aircraft development programs, organizations tasked with the development of long lead items, such as the aircraft engines or the landing gears, have to start working a long time before mature aircraft requirements are made available to them.

Such organizations traditionally have a number of options regarding how they could deal with such a situation: (1) they could wait and only start working after they have received their validated input requirements from the top level; (2) using previous experience and a number of assumptions, they could start working at their level, without exactly knowing what their input requirements will be; or (3) they could ask for preliminary versions of their input requirements in order to start their work based on those. 
The above options have some serious drawbacks. Option (1) means that these organizations will work on a sound basis, but won't have much time to develop their long lead items, i.e. this option could result in delays for the overall aircraft program. Option (2) would be much riskier and prone to corrective rework, which could lead to unplanned costs and delays. Also, this would potentially lead to conservative rather than innovative design solutions. Option (3) would imply a high degree of risk, too, as the development efforts would be based on immature input requirements, invariably leading to high levels of corrective rework, which in turn will lead to unplanned costs and delays, as well as conservative rather than innovative design solutions. In addition, even as requirements has been decomposed and validated, there still remain a risk of misconception and delay due to the simple fact that requirements need to be interpreted, and their context understood. Since aircraft requirements are typically cascaded several times before being available by sub-system manufacturers, retaining their governing intent and rationale is by nature difficult.

The objective of the paper is to present a Value Driven Design (VDD) methodology, developed within an EU FP7 project in aeronautics named "Collaborative and Robust Engineering Using Simulation Capability Enabling Next Design Optimisation" (CRESCENDO, 2012), which targets the need for design iterations to be executed in the very beginning of an aircraft development program. The aim of the proposed methodology and associated support tools is to support the requirements establishment process and strengthen the value contribution focus into the development organization. This is expected to reduce development risks and corrective rework considerably.

\section{The Value-Driven Design Methodology}

The Value-Driven Design (VDD) methodology proposed in the paper aims to complement traditional systems engineering (SE) processes (INCOSE, 2011) by using the concept of value for early design concepts generation and selection. The methodology is inspired by previous VDD approaches proposed in literature (Castagne et al., 2009, Collopy and Hollingsworth, 2011) and aims to enable what-if assessment loops to be executed at all levels of the supply chain in the very early stage of the aircraft design process, well before detailed requirements are made available by the aircraft manufacturer. Compared to previous work in INCOSE (Roedler et al., 2005), the proposed framework adopts an information driven approach that link customer expectations to characteristics and enabling value fulfillment as drivers for design within an extended enterprise through iterations.

The Value Creation Strategy (VCS) is the entity (or document) that carries preliminary design information across the supply chain partners, and enables their teams working on long lead items to initiate the development work earlier than what happens today. A VCS is a description of a specific context for a project. Initially, it includes a set of rank-weighted needs that have to be satisfied for identified stakeholders or customer profiles. In later iterations it also encompasses a list of rank-weighted objectives with corresponding measurement criteria and a set of Value Drivers (VDs). VDs indicate key engineering characteristics given a specific VCS (i.e. for a specific stakeholder profile and context). They represent solution directions that seem to have a significant influence on the value perceived by the customer (or by the stakeholders) in a given problem context. VDs are not attached to a target value or function, but they tend to result in measurable objectives and later, based on these, in requirements. The VDD methodology follows a series of linked activities, tailored to facilitate iterations (Figure 1): 


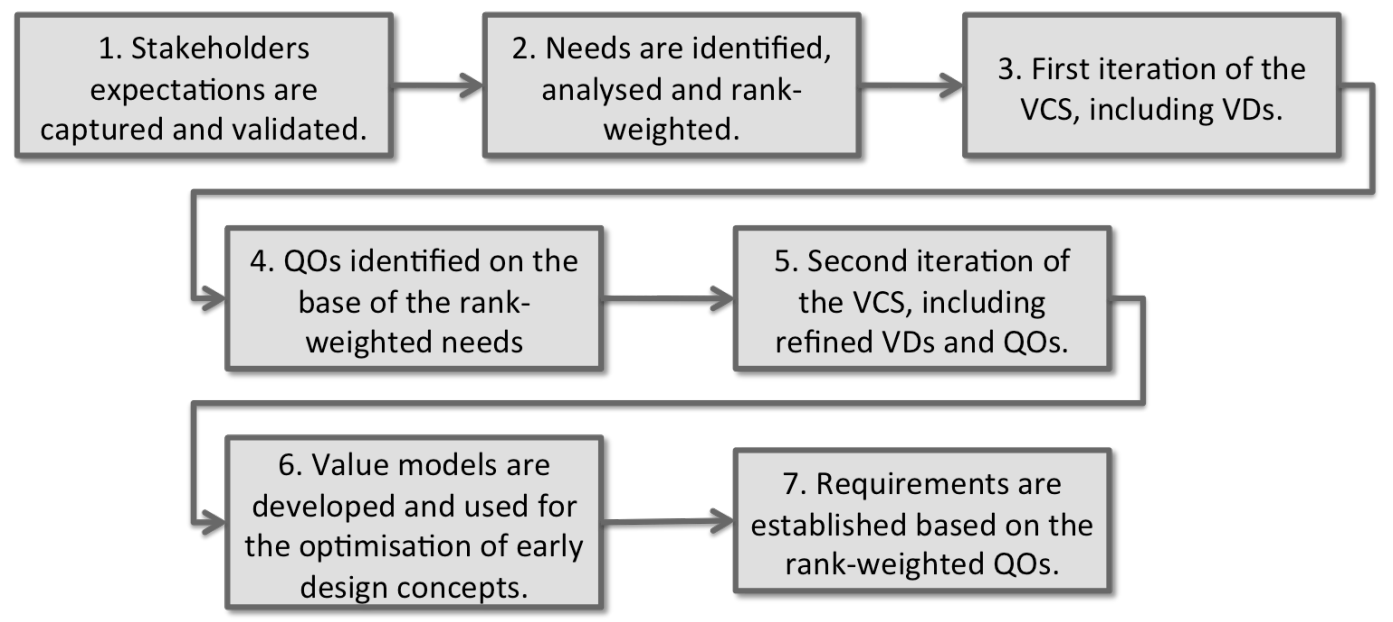

Figure 1. High level Value-Driven Design process

The first step (1) deals with capturing and validating stakeholder expectations, which are then organized and interpreted in terms of needs, which are further rank-weighted on the base of their importance for a given context (2), such as the low-cost carrier market segment that the aircraft program aims to target. A first VCS is developed for the given context (3) and contains a context description, the rank-weighted needs for the context and a list of suggested VDs (essentially, system characteristics that are expected to particularly contribute to stakeholder value). Later in the development cycle, a hierarchy of Quantified Objectives (QOs) with specific target values or target functions is identified for each rank-weighted need (4). Given this input, a second VCS iteration can be developed (5), containing a refined description of the context and of the VDs for the study. It also contains an optimum selection of QOs that represent design concepts or solutions that have been recognized to satisfy the targeted stakeholders' needs. This loop helps to increase confidence that the business case in terms of the retained set of objectives is sound, and that the proposed concepts or solutions are achievable.

The VCS is then used as input to perform optimization of early design concepts at different levels of the supply chain (6), using a range of approaches as described in the following sections. Once the most value adding design concepts are identified, requirements are established based on the rank-weighted QOs (7) and can be communicated in a third iteration VCS, which summarizes the updated context information and makes reference to the validated requirements.

\section{The extended enterprise view of the VDD methodology}

Within the extended enterprise, the VCS can be collaboratively built much earlier than is the case where merely a traditional approach to Requirements Management is deployed (see Figure 2), and can be more easily communicated from one level to the next (Monceaux and Kossmann, 2012). The aircraft level VCS description, which contains high-level information on frame, power-plant and cabin design, is initially cascaded down to engine level. The engine manufacturer uses the VCS, together with other information, to rank-weight engine needs. The description of the rank-weight engine needs is added to the VCS, and is cascaded further down to the engine sub-system level, and so on. 


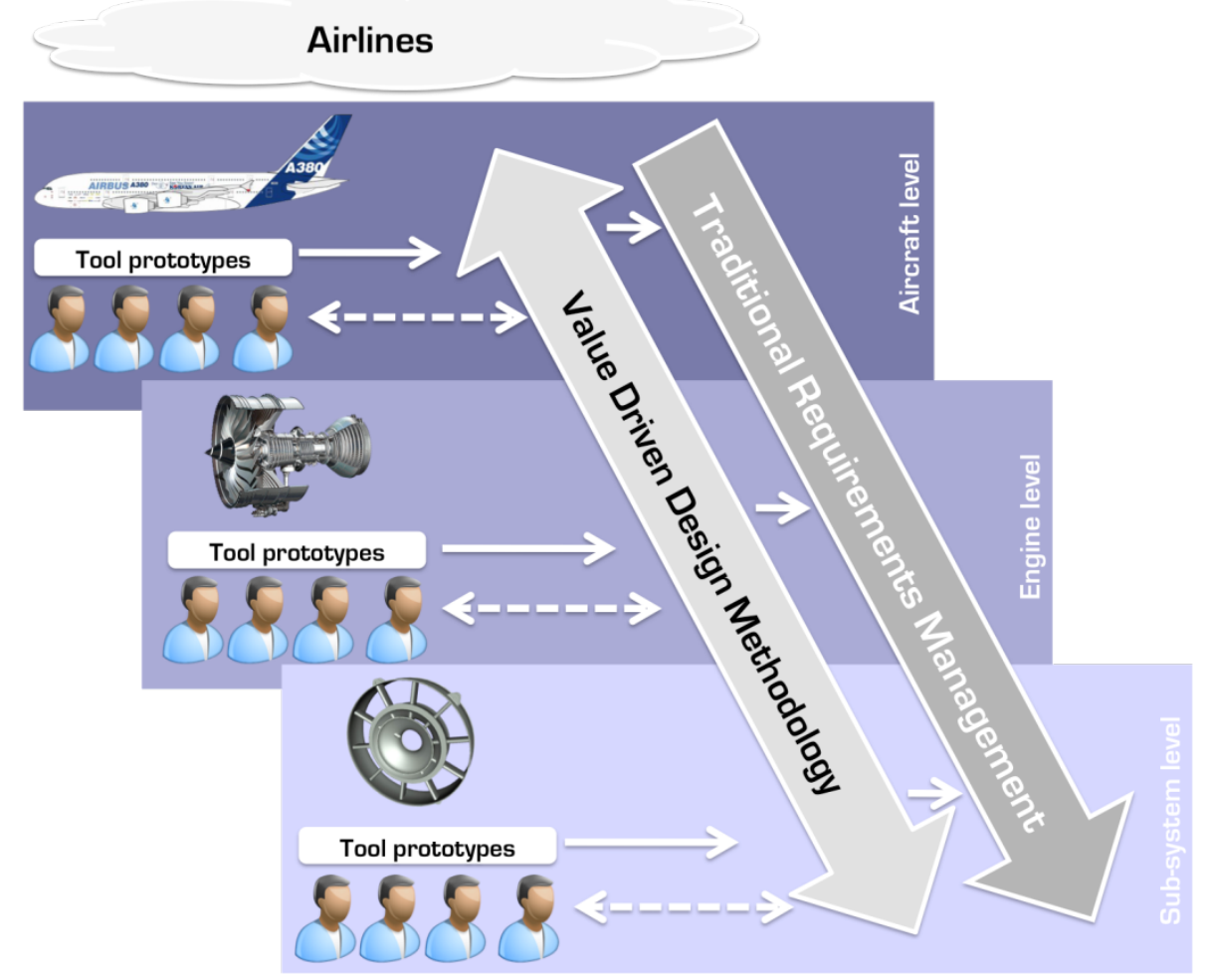

Figure 2. VDD enhances traditional RM within the extended enterprise

Figure 3 and Figure 4 exemplify how two aircraft level needs are cascaded down in the supply chain and how they can be re-elaborated locally by the partners.

- Need 1: 'To be known for passengers first.'

- Need 2: 'To be the most fuel efficient.'

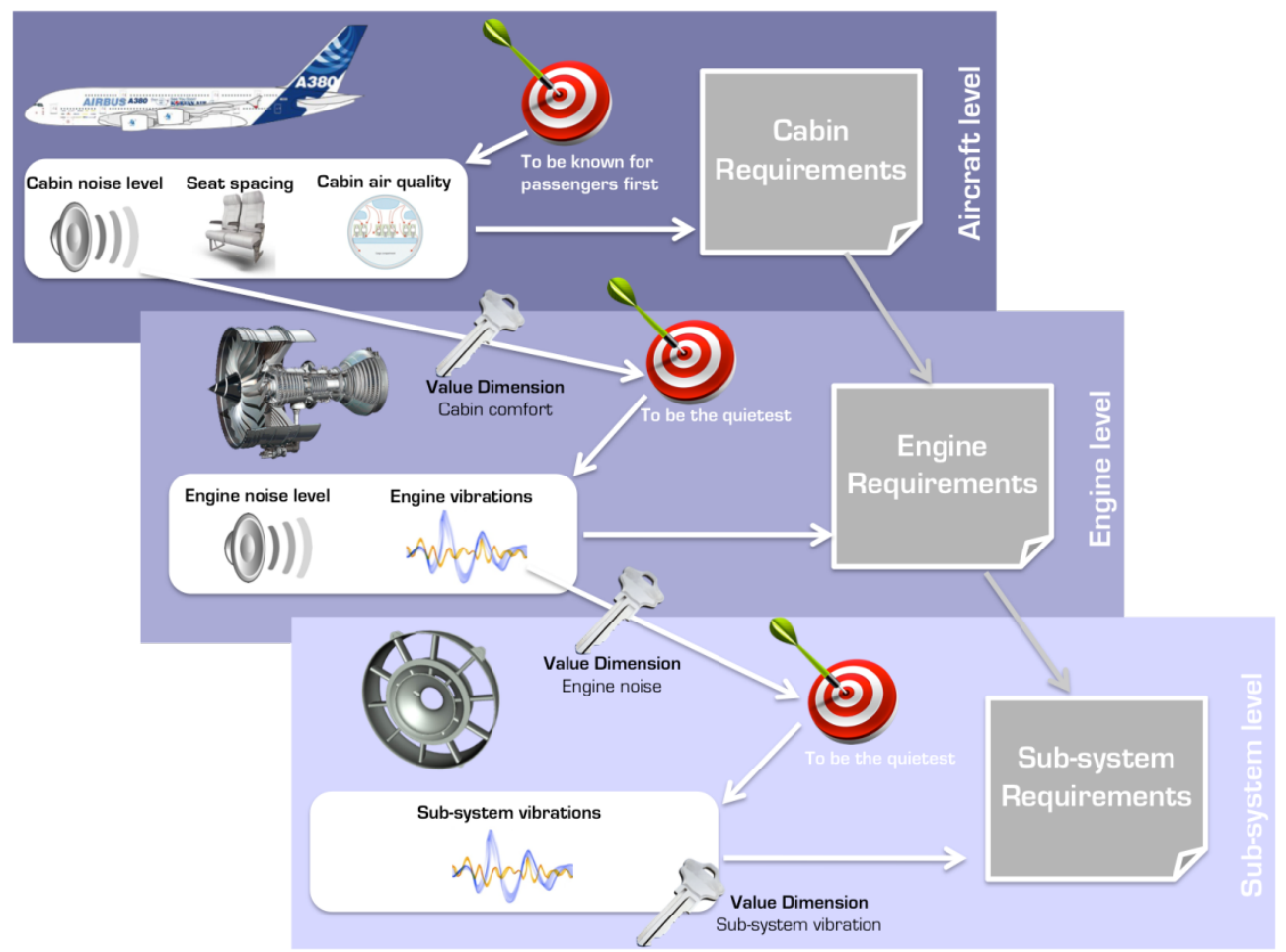

Figure 3. Translating Value Drivers from one level to the next (Comfort) 
In Figure 3, the need 'to be known for the passengers first' is translated at aircraft level in three main VDs, such as 'cabin air quality', 'cabin noise level', and 'seat spacing'. Of course, there could be additional value drivers such as 'cabin lighting', 'vibration level', or more detailed value drivers, e.g. 'leg room' and the 'texture of seat surfaces'. The initial selection of the value drivers at $\mathrm{A} / \mathrm{C}$ level can be communicated in a first iteration of the VCS to the engine level. The 'cabin noise level' VD can be translated, into more detailed drivers at engine level, such as 'engine noise level' regarding air transmitted noise, and 'engine vibrations' regarding structure transmitted noise. Furthermore, they are cascaded one level down and further characterized by the sub-system manufacturer.

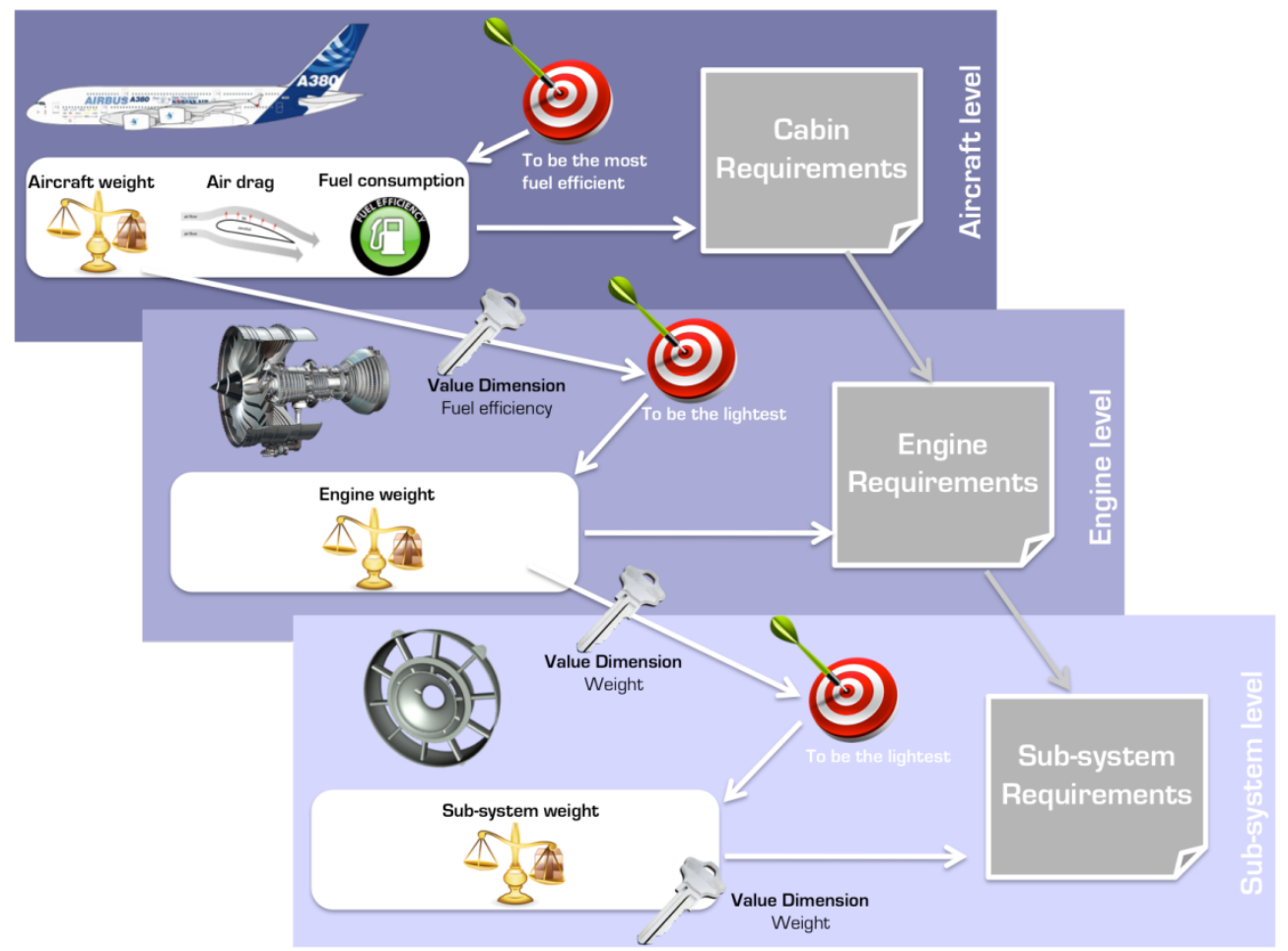

Figure 4. Translating Value Drivers from one level to the next (Fuel efficiency)

In Figure 4 the need 'to be the most fuel efficient' is translated at aircraft level in three main VDs, such as 'aircraft weight, 'air drag', and 'fuel consumption'. Also in this case, the VDs can be re-used from one level to the next in the cascade. This initial selection of VDs at aircraft level can be communicated in a first iteration of the VCS to the engine level. The 'aircraft weight' driver can be related to 'engine weight', and, further down in the supply chain, to 'sub-system weight'.

\section{The VDD methodology in detail}

Figure 5 provides a high level view of the simplified VDD process across three example levels of development in the extended enterprise, the aircraft, engine, and sub-system level. These levels are simplified, for instance, the aircraft level comprises at least the aircraft program, the aircraft and the cabin and cargo levels. These levels have been selected to demonstrate how the VDD process could work within the extended enterprise. 


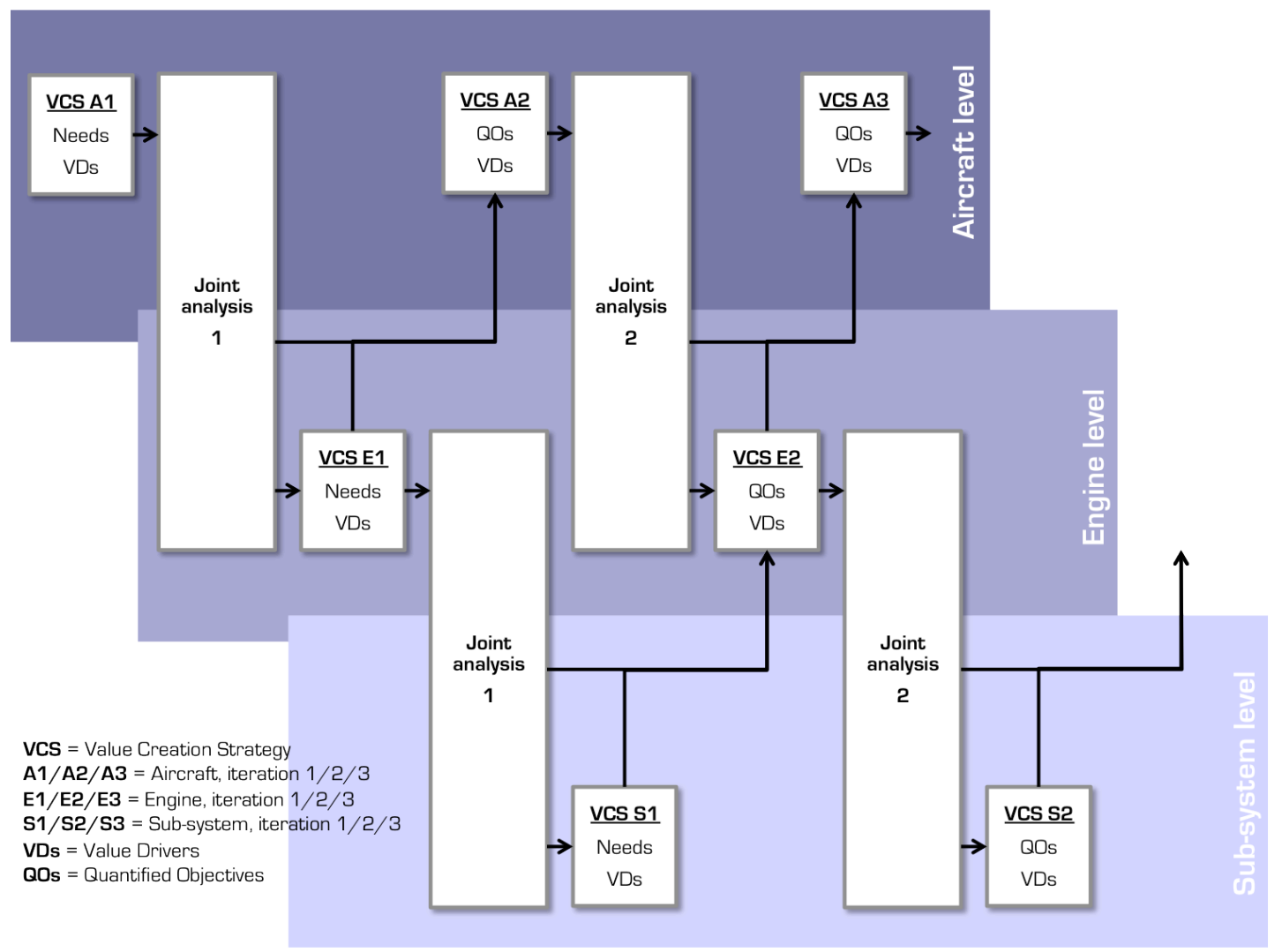

Figure 5. The VDD process within the Extended Enterprise (simplified)

The VDD process is built around several iterations of VCS at each concerned level, with joint analysis phases between two interfacing levels between each such iteration. In the following, the generic VDD iterations are explained in more detail.

First iteration at aircraft level

- Based on captured, analyzed and validated expectations as well as other relevant context knowledge, a list of external and internal high-level needs is formulated for a given context.

- These needs are analyzed, validated and rank-weighted with the relevant stakeholders (and a suitable value dimension is selected as an attribute of each need).

- Initial value drivers are identified for each need, as something which is expected to have a significant impact on the achievement of the corresponding stakeholder need.

- A first iteration VCS is formulated, containing a free context description, the list of rank-weighted needs and the initial value drivers.

First iteration at engine level

- Based on captured, analyzed and validated expectations as well as other relevant context knowledge, in particular the first iteration VCS at aircraft level, a list of external and internal high-level needs at engine level are formulated for the given context.

- These needs are analyzed, validated and rank-weighted with the relevant stakeholders (and a suitable value dimension is selected as an attribute of each need).

- Initial value drivers are identified for each need, as something which is expected to have a significant impact on the achievement of the corresponding stakeholder need. These value drivers are likely to at least partly differ from the value drivers defined at 
the aircraft level.

- A first iteration VCS is formulated, at the engine level, containing a free context description, the list of rank-weighted needs and the initial value drivers.

First iteration at engine sub-system level

- Based on captured, analyzed and validated expectations as well as other relevant context knowledge, in particular the first iteration of the VCS at engine level, a list of external and internal high-level needs are formulated for the given context.

- These needs are analyzed, validated and rank-weighted with the relevant stakeholders (and a suitable value dimension is selected as an attribute of each need).

- Initial value drivers are identified for each need, as something which is expected to have a significant impact on the achievement of the corresponding stakeholder need. These value drivers may differ from the value drivers defined at the engine level.

- A first iteration VCS is formulated, at the sub-system level, containing a free context description, the list of rank-weighted needs and the initial value drivers.

Second iteration at aircraft level

- Based on feedback from the joint analysis and in light of the first iteration of the VCS at engine level, as well as more detailed value modeling activities at aircraft level, relevant quantified objectives are elaborated that are expected to jointly satisfy the stakeholder needs.

- This set of quantified objectives will be subject to specific value modeling activities by means of suitable methods and tools, in order to optimize the overall value of the entire set of quantified objectives.

- The initial set of value drivers is either confirmed or modified in light of the above value modeling activities.

- A second iteration VCS is formulated at aircraft level, containing a refined free context description, the list of rank-weighted needs, the confirmed or updated value drivers, and the set of rank-weighted quantified objectives.

In summary, the VDD methodology comprises of a series of generic activities that can be used at any level or within any organization. To be able to use the VDD in practice, the concept needs to be modeled and supported by an information model so ensure consistent use. In CRESCENDO - the VDD methodology was described in the Behavioral Digital Aircraft (BDA) - model. The BDA information model is a core result from CRESCENDO encompassing not only the VDD aspects, but support for several different engineering oriented business processes (CRESCENDO, 2012). It has enabled the use of information communication and integration using web services to access data and simulation models.

\section{VDD Methods and Supporting Tool Prototypes}

The VDD methodology needs to be supported by methods and tools. In the following sections we present first how to formally capture and issue a VCS. Secondly, we present how to use modeling and simulation tools to assess the value contribution. A method to use the VCS information for concept down selection is briefly described and finally we show how to assess value on sub-system level technologies.

\section{The building and sharing of a Value Creation Strategy}

In our approach, Value Drivers (VDs) are used to capture and make stakeholders needs explicit. First stage of this process takes place after that needs have been captured by using 
customer focus groups or by other methods. Needs are analyzed and validated with the relevant stakeholders and a suitable value dimension is selected as an attribute to classify each stakeholder need. Initial value drivers are identified for each need, as something which is expected to have a significant impact on the achievement of the corresponding stakeholder need. Table 1 shows a set of example data from the aircraft level linking captured stakeholder needs with their dimensions and associated value drivers.

Table 1: Aircraft level Need - Value dimension - Value drivers

\begin{tabular}{|c|c|c|}
\hline Stakeholder needs & Value Dimensions & Value Drivers \\
\hline $\begin{array}{c}\text { The AC needs to be available for } \\
\text { operational use as predicted and } \\
\text { planned. }\end{array}$ & $\begin{array}{c}\text { Maintenance } \\
\text { (scheduled and } \\
\text { unscheduled) }\end{array}$ & $\begin{array}{c}\text { Accessibility } \\
\text { Inspectability } \\
\text { Reparability }\end{array}$ \\
$\begin{array}{c}\text { The expected fuel consumption needs to } \\
\text { be perceived as the lowest. }\end{array}$ & Fuel Consumption & A/C resistance \\
& & $\begin{array}{c}\text { Electricity consumption } \\
\text { Energy consumption }\end{array}$ \\
Engine fuel consumption
\end{tabular}

In order to demonstrate the approach, we establish one or several explicit and rank-weighted sets of VDs to consider in the context of a project or a study and we represent them as structured data usable for a further value modeling stage using the CODA method (see the following section for the CODA methodology). To that purpose, a survey process involving a panel of interviewees is used to collaboratively elicit and rank the VDs. A web application (see Figure 6) allows capturing and structuring the stakeholder needs through a succession of votes regarding successively who are the key stakeholders, which of their value dimensions and VDs (in relation with needs) are judged most relevant, and finally what is the VDs' dependency with regards to customers' (airlines') profiles. Several VCSs can output from this 
process; a VCS being a relevant and contextualized set of criteria against which we can (later on) assess and down-select alternative aircraft concepts.

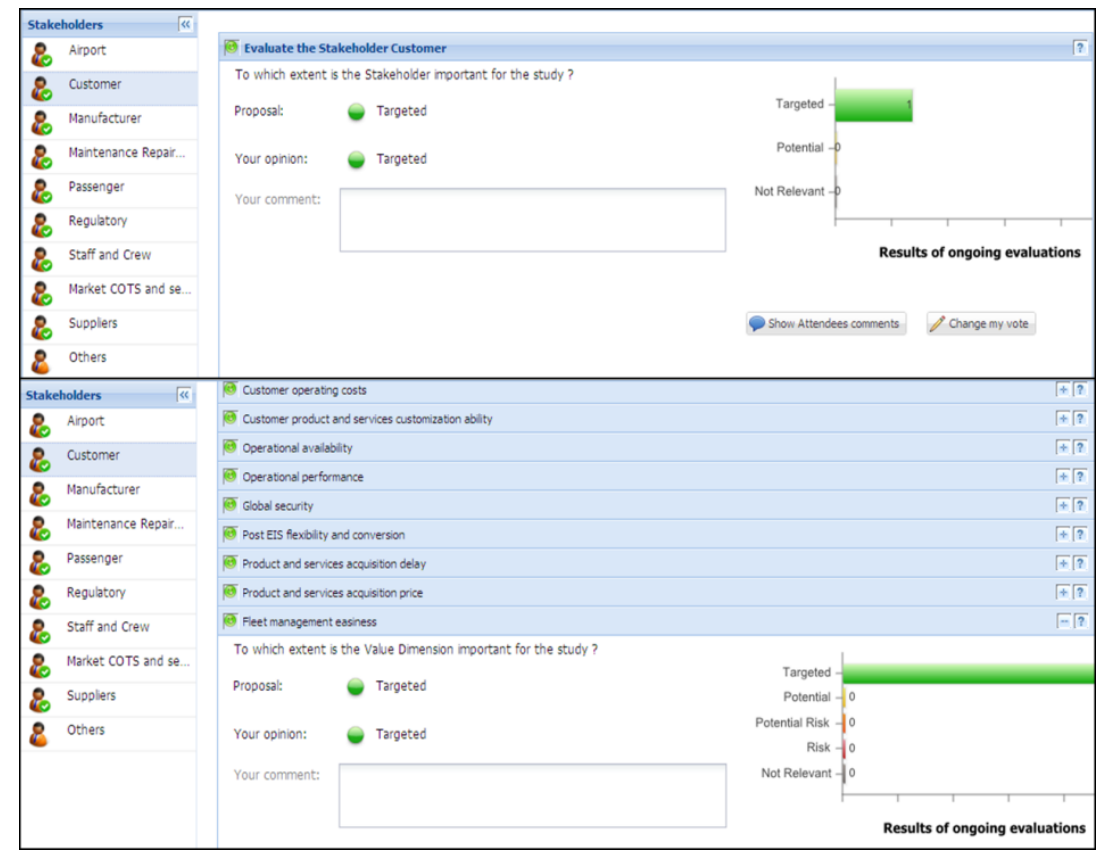

Figure 6. Voting tool

The resulting votes file is then exchanged through the BDA web services, as a set of structured data that can be managed in relation with its context (applicable program, trade studies, etc.) in order to facilitate the sharing of value information between people and the interoperability between tools. The BDA web services allow creating and executing cross-company workflows whilst protecting company intellectual properties.

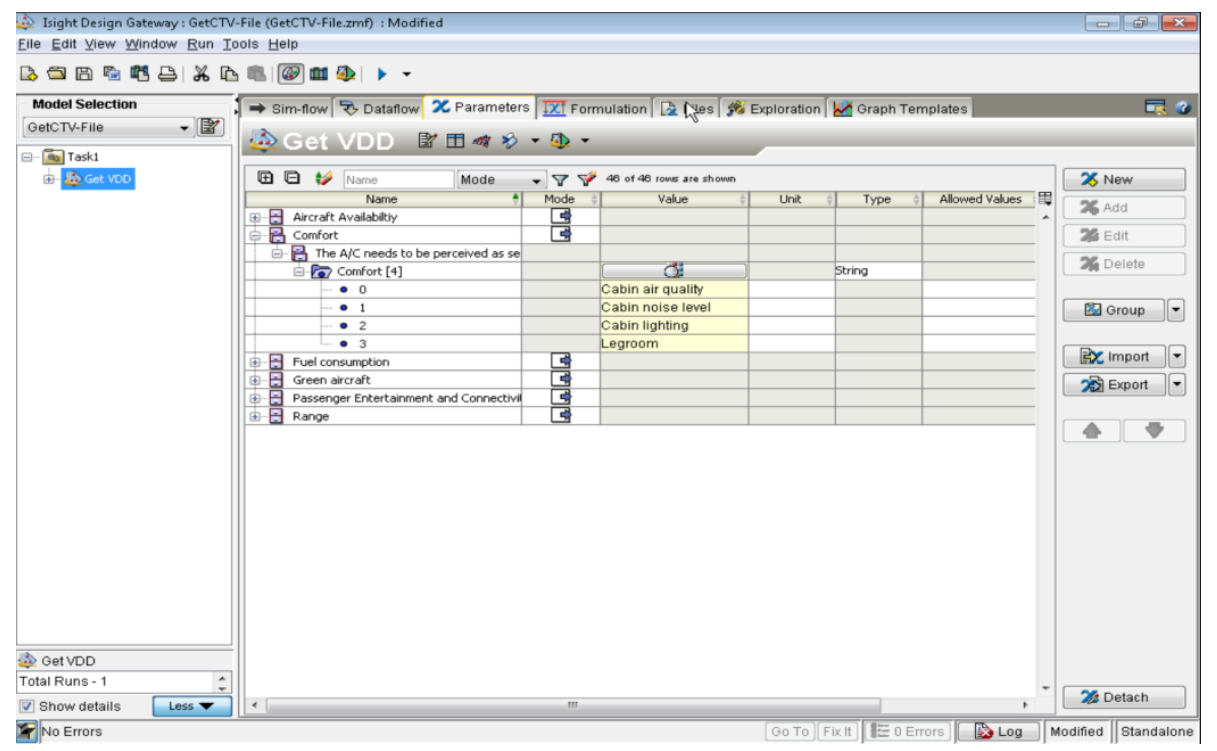

Figure 7. VCS structured data

As examples of sharing services, we have demonstrated how the VCS data is imported and managed, at some given partner's place, using the local Product Lifecycle Management (PLM) tool, ENOVIA V6. We also demonstrated how the VCS data is analyzed and transformed using a process flow execution engine, Isight (see Figure 7), in order to 
automatically generate a prefilled "Need" part of a 'blank' CODA template, where the conceptual work to assess design alternatives using ranked weighted value information is managed. The approach stores the design rationale so that the need data used in the VCS can be used and shared.

\section{The assessment and simulation of value}

In order to demonstrate a VDD approach in the concept down selection process of alternative engine architectures, a conventional aero-engine and a more electric engine are compared in terms of surplus value (SV) they generate during their operational life. Considering an ever increasing demand for in cabin electric power from fare paying passengers, the aircraft manufacturer asks for more electrical power options from the aero-engine manufacturer. In this scenario, the aero-engine manufacturer has to assess different engine architectures by using performance, cost (unit, maintenance, life-cycle, etc.), SV, and design merit or 'goodness'. The most straightforward implementation on a conventional aero-engine is to replace the mechanical oil system with an electrical oil system by using electric oil pump and scavenge system and electric accessory gear box oil scavenge system. These changes would not impose any design change on the aircraft design, but they would eliminate the daily oil top-up maintenance on the engines, thus reducing the overall maintenance cost and increase the SV generated during their operation. These models are implemented in Vanguard Studio (Vanguard, 2012) and a schematic view of them is presented in Figure 8. Preliminary simulations for conventional and more electric aero-engine architectures resulted in a decrease in the whole engine unit and maintenance cost and thus an increase in the SV of an aircraft fleet using more electric engine.

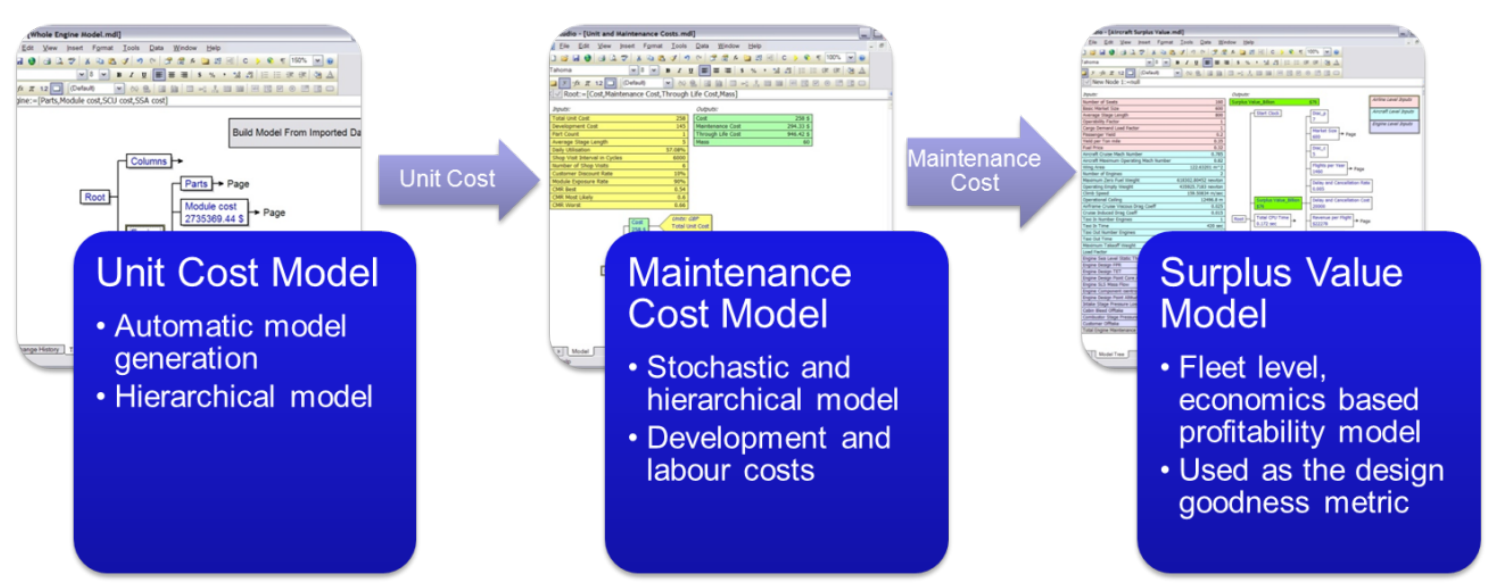

Figure 8. Schematic view of the unit and maintenance cost, and SV models

\section{The concept down selection using CODA}

Once the VCS has been initiated, the conceptual development work can be started, and the following questions are raised - What conceptual product alternatives contribute most to the desired VCS? Can we derive a "design merit" that represent the value contribution of conceptual alternatives, and use this for down selection? The Concept Design Analysis (CODA) methodology (Woolley et al., 2000, Woolley et al., 2001, Feneley et al., 2003) was used and refined to enable such task. This methodology involves the following steps:

1. Identify customer needs (surveys, customer focus groups, etc.) as expressed in the VCS

2. Find percentage weights of each customer need (a binary weighting model is used, but other methods can also be utilized). 
3. Identify engineering characteristics and their lower and upper limits that have an effect on any number of customer needs.

4. Decide on the mappings between customer needs and engineering characteristics. (See Figure 9)

a. Is there a strong, medium, weak or no correlation?

b. Decide if there is a minimization, maximization, or optimization relationship between customer need and engineering characteristic.

c. What is the neutral point where the customer satisfaction level is $50 \%$ ? Or for optimization relationship, what are the optimal and tolerance values?

d. Calculate the individual merit value.

5. Repeat step 4 for each customer need and engineering characteristic. Note that there may be no relationship between some of them.

6. Calculate the overall design merit.

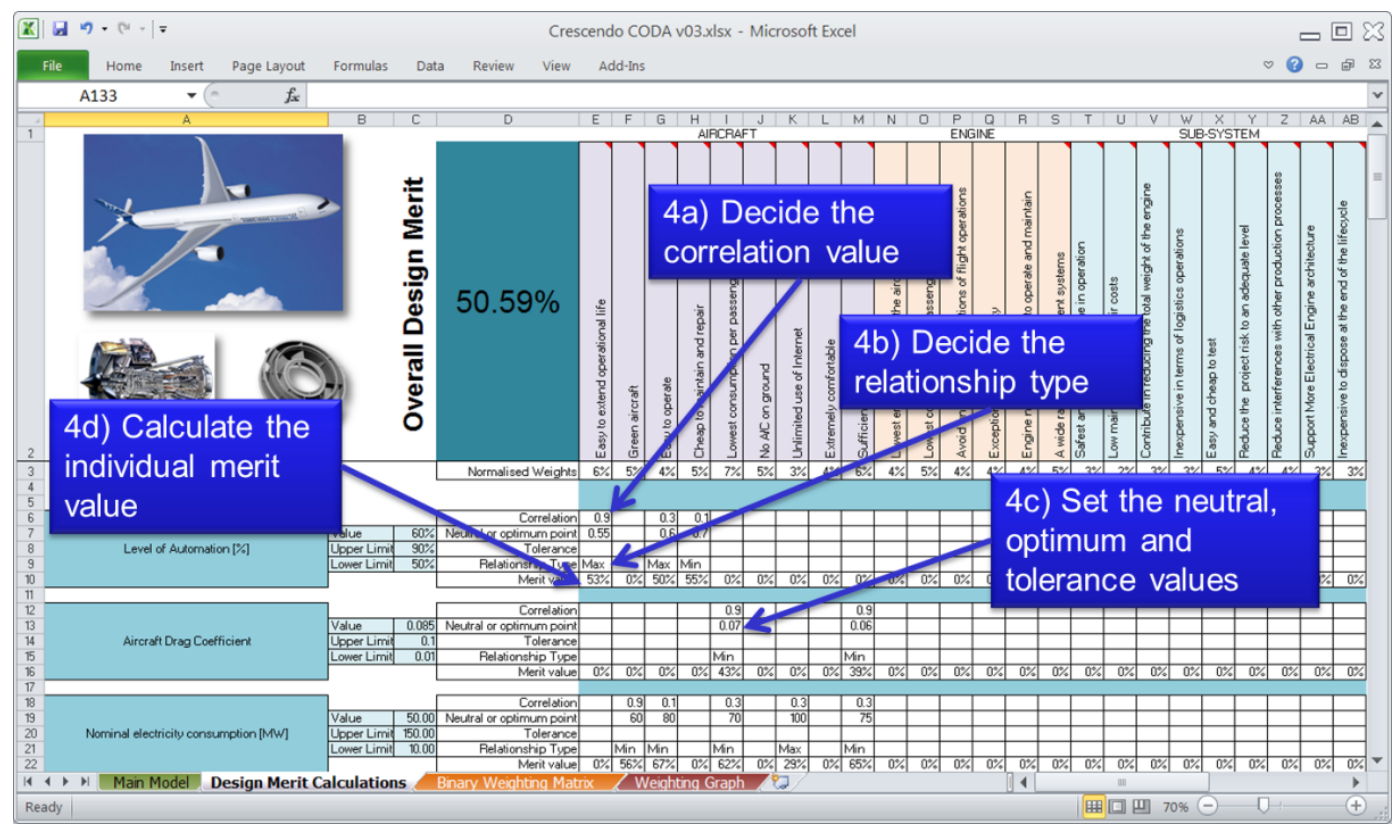

Figure 9. An excerpt from the CODA calculation sheet

In general, there are several possible ways to define and assess value, each with their advantages and limitations. The CODA model presented here uses a relative value indicator which can be seen as a percentage scale describing design merit or overall customer satisfaction level. More economic based models utilizing surplus value as the objective function for design assessment (Collopy et al., 2009, Cheung et al., 2012) and utility theory based models for conflicting customer needs (Zhang et al., 2012a, Zhang et al., 2012b) exist in the recent literature.

\section{Assessment of value at sub-system level}

At sub-system level, methods and tools are needed to use the VCS information to benchmark conceptual component/technology alternatives on the base of their "goodness" at system level, and to eventually adjust early design decisions. EVOKE (Early Value Oriented design exploration with KnowledgE maturity) elaborates on the CODA matrix and adapts it to the purpose of understanding which concept contributes most to the desired VCS.

EVOKE produces, as CODA, a design merit score, which is calculated across a list of sub-system value drivers that are believed to reflect the system-level contribution of a design concept. To do so, EVOKE employs 3 matrices: the Weighting Matrix (WM), which cascades down the system-level VCS to sub-system (local) value drivers, the Input Matrix (IM) which 
gathers information about the characteristics of each design alternative under consideration, and the CODA matrix, which renders the Design Merit score. EVOKE has been demonstrated using an aircraft engine intermediate compressor case (IMC) as reference design. Figure 10 shows the results of the assessment performed on two IMC concepts, on the basis of the 10 value drivers used in the IMC study.

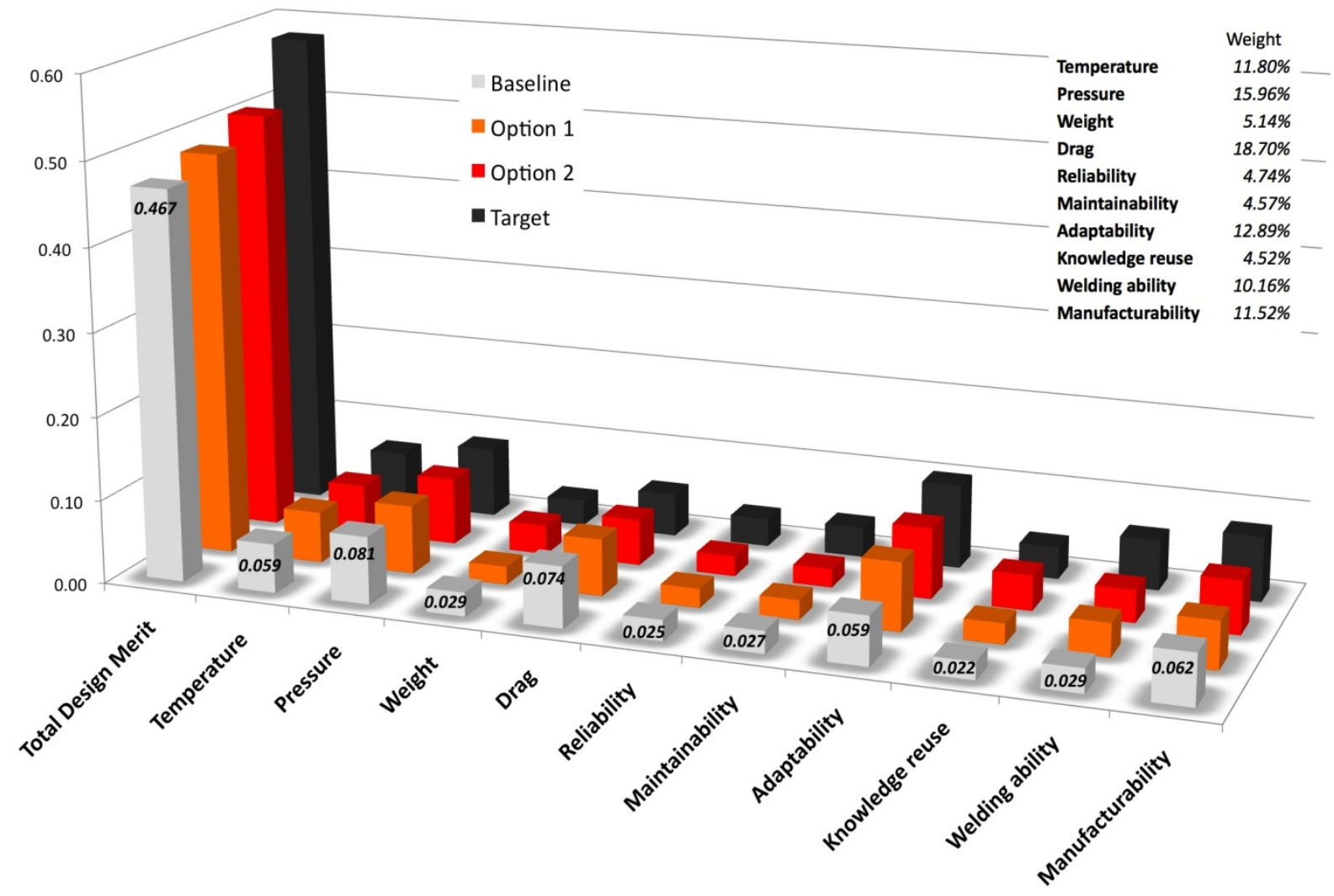

Figure 10. Results of the CODA matrix

Weighting matrix. After having defined a list of relevant sub-system (local) VDs for the study, the WM is used to rank-weight them on the basis of information from the VCS. Different VCSs emphasize different aspects of the local solution. A VCS that prioritizes fuel consumption at engine level emphasizes local drivers such as temperature and pressure in the component, which are less crucial when focusing on passenger comfort or noise. The weighted sub-system VDs are used as input in the CODA matrix.

Input matrix. The design team needs then to define the high-level features of sub-system alternatives that will be assessed using CODA. Using reference designs as benchmarks (such as a baseline design and a target one), the design team creates a number of alternative concepts for the IMC, which differ in terms of shape, material, reuse of technology, production lead-time or maintainability characteristics. The characteristics of each concept are formalized in a list of so called Quantified Objectives, which are also inputted in CODA.

CODA matrix. CODA is used to evaluate the fulfillment of a sub-system value driver given the list of QOs for each design option. In the same way as described in the previous section, CODA renders a total design merit for a design by aggregating the merits for each VD.

Knowledge Maturity. EVOKE matrixes are largely built on assumptions and populated with figures characterized by different levels of confidence (i.e., for known technologies the input data can be widely known and assessed, for radically innovative solutions they might just be an educated guess). A Knowledge Maturity (KM) model is used then to provide a feedback to the designers about the reliability of the value analysis results. A $K M$ score from 1 (lowest 
maturity) to 5 (highest maturity) is associated to each QO, correlation and function in the EVOKE matrixes. They are then aggregated in an overall KM score for each design. KM helps to orient the decision makers' choice by highlighting the assessment that is based on the most mature knowledge (e.g., Option 1 in Figure 10).

The main reason for not using CODA As-Is when working at component level, is that here designers do not work directly with end user and customer needs (e.g., 'cabin noise level'), rather their receive as input a list of dimensions and drivers that interpret them from the standpoint of the system provider (e.g., 'engine noise level'). The Weighting and Input Matrix reflect the need for translating such interpretations in term that are more meaningful at sub-system level, while Knowledge Maturity captures the uncertainty in the information caused by the cascading process. The purpose of EVOKE, as well as CODA, is not to have an exact measurement; rather it is to have a common denominator that triggers the debate around the team members' perception of value contribution, especially when opinions differ. In this spirit, the work has also brought to the development of an approach that uses color-coded CAD models to display the results of the value assessment activity, and in this way improve communication and collaboration in the within and across the design team. The approach has been implemented in SIEMENS NX environment and tested both in industry and in design sessions with students (Bertoni et al., 2012).

\section{Conclusion and discussion}

The Value Driven Design methodology and associated methods and tools presented in the paper have been designed with the objective to complement and strengthen the requirements establishment process and introduce a more explicit way to focusing end user value contribution into product development. In particular, the ability of align work methods within an extended enterprise require a common way of working. Consequently the methodology has been mapped and represented into a BDA model - an information model enabling efficient sharing of information between systems and organizations.

This approach of communicating iterations of increasingly detailed VCS between different levels of the extended enterprise, allows early conceptual work that is directed towards areas of highest added value in a given context. In case some privacy issues arise because certain needs and stated preferences in the VCS reflect internal business strategy, the key elements of a VCS can still be communicated, namely the selection of rank-weighted needs or QOs, with their classifying value dimensions and associated value drivers. Also, the very process of going through iterations of VCS generation will greatly enhance the efficient and effective development of requirements that are directly driven by stakeholder perceived value. The identified risks and issues related to early development work prior to having validated input requirements may be effectively mitigated, thereby reducing overall development times and costs, while improving quality and stakeholder perceived value.

The Value Driven Design Methodology strengthens the requirement establishment process by emphasizing the maturation of the requirements through iterations within the developing enterprise. Secondly, VDD emphasizes the value focus and ensures it to remain into the sub-sequent development work. The VDD has been shown to be supported by several methods and tools, such as the CODA model for concept down selection and the EVOKE for comparing technologies and including maturity assessments. Importantly, the VDD methodology formalizes associated ontologies and establishes a common reference information model enabling coherent implementation of information systems using standardized web services. 
The VDD has been developed to a degree where it has been shown to be practical, and contribute to the early phases of development, yet there are several areas for improvement and maturation. For extended enterprise collaboration, the opportunities and potential benefit is significant, but so are also the challenges. Examples include Configuration Management of Value Generation data and the apparent fact that as several VCS's are created there is a need to handle conflicting content of VCS's. The VDD methodology should be integrated with related SE processes rather than having several independent processes in parallel. The methodology in particular, but also Value Generation in general is multi-disciplinary and therefore has to be considered in the comprehensive context of Systems Engineering rather than in isolation.

Further areas include management of information maturity evolution, the role of strategic partnerships within the extended enterprise, implementation/deployment strategies and VDD in the extended enterprise as an optimization problem.

\section{Acknowledgement}

The research leading to these results has received funding from the European Union Seventh Framework Programme (FP7/2007-2013) under grant agreement $n^{\circ} 234344$ (www.crescendo-fp7.eu/).

\section{References}

BERTONI, M., BERTONI, A. \& ISAKSSON, O. 2012. Experiences with Value Visualisation in preliminary design: results from an aero-engine component study. $1 s t$ International Conference on Through-life Engineering Services. Cranfield, UK.

CASTAGNE, S., CURRAN, R. \& COLLOPY, P. 2009. Implementation of value-driven optimisation for the design of aircraft fuselage panels. International Journal of Production Economics, 117, 381-388.

CHEUNG, J., SCANLAN, J., WONG, J., FORRESTER, J., ERES, H., COLLOPY, P., HOLLINGSWORTH, P., WISEALL, S. \& BRICENO, S. 2012. Application of Value-Driven Design to Commercial Aero-Engine Systems. Journal of Aircraft, 49, 688-702.

COLLOPY, P., FORRESTER, J., WISEALL, S., HOLLINGSWORTH, P., SCANLAN, J., ERES, H., CHEUNG, J. \& HOPTROFF, J. 2009. Value driven design - Scoping and feasibility study (Workshop 1).

COLLOPY, P. D. \& HOLLINGSWORTH, P. M. 2011. Value-Driven Design. Journal of Aircraft, 48, 749-759.

CRESCENDO. 2012. Collaborative and Robust Engineering using Simulation Capability Enabling Next Design Optimisation [Online]. Available: http://www.crescendo-fp7.eu// 14 May 2012].

FENELEY, R. C. L., PARKIN, J., SCANLAN, J. \& WOOLLEY, M. 2003. Developing alternative devices to the long-term urinary catheter for draining urine from the 
bladder. Proceedings of the Institution of Mechanical Engineers Part H-Journal of Engineering in Medicine, 217, 297-303.

INCOSE 2011. Systems Engineering Handbook v3.2.2.

MONCEAUX, A. \& KOSSMANN, M. 2012. Towards a Value-Driven Design Methodology - Enhancing Traditional Requirements Management Within the Extended Enterprise. 22nd Annual INCOSE International Symposium. Rome, Italy.

ROEDLER, G. J., JONES, C. (Eds.), Technical Measurement, 2005, INCOSE-TP-2003-020-01

VANGUARD. 2012. Vanguard Software Website [Online]. Available: http://www.vanguardsw.com/.

WOOLlEY, M., SCANLAN, J. P. \& EVESON, W. 2000. The use of Formal Design Techniques in the Development of a Medical Device. IMechE International Engineering Design Conference 2000 Brunel University, Uxbridge, UK.

WOOLLEY, M., SCANLAN, J. P. \& EVESON, W. 2001. Optimising the Development of a Medical Device Using Formal Engineering Design Techniques and the CODA-System. ICE 7th International Conference on Concurrent Engineering. Bremen, Germany.

ZHANG, X., AURIOL, G., ERES, H. \& BARON, C. 2012a. A prescriptive approach to qualify and quantify customer value for value-based requirements engineering. International Journal of Computer Integrated Manufacturing, 1-19.

ZHANG, X., AURIOL, G., ERES, H., BARON, C. \& KOSSMANN, M. 2012b. Understanding Airlines' Value Perceptions For Value-Based Requirements Engineering Of Commercial Aircraft. 22nd Annual INCOSE International Symposium. Rome, Italy.

\section{Biography}

Ola Isaksson is a senior company specialist in product development at GKN Aerospace Engine Systems, Sweden, where he has been working since 1994. He is also an adjunct professor at Luleå University of Technology in Functional Product Development. He has a MSc in Mechanical Engineering, a $\mathrm{PhD}$ in Computer Aided Design and an associate professorship in functional product development. Ola's work and research experience concern methods, tools and processes include areas such as computational support for product development, knowledge based engineering, value based engineering, systems engineering and product-service system development. He led the Value Driven Design work in the CRESCENDO project.

Mario Kossmann (CSEP) is an experienced Systems Engineer and Capability Integrator for Airbus, having previously worked for Blohm \& Voss as Systems Engineer, Technical Manager and Consultant in Services Marketing. He has served as a naval officer with the German and French navies, and was awarded an MEng in Aerospace Technology from the University of the Federal Armed Forces in Munich (Germany), an MBA from the University 
of Warwick (UK) and a Ph.D. in Requirements Engineering from the University of the West of England. Mario is also a certified Project Manager and a Certified Systems Engineering Professional.

Marco Bertoni is Associate Professor in mechanical engineering at Blekinge Institute of Technology in Sweden. His research focuses on the concept of value to complement traditional Requirements Engineering process, and on engineering knowledge management approaches to support early stage decision-making in design. He has experience in European and national research projects in the domain of automotive and aerospace.

Hakki Eres is a senior research fellow at the Faculty of Engineering and the Environment, University of Southampton. He received his BSc and $\mathrm{MSc}$ degrees in Mechanical Engineering from Boğaziçi University, Turkey in 1990 and 1993, respectively. He obtained his PhD degree in Mechanical Engineering from University of Delaware, USA in 1998. His research interests are in the fields of numerical solutions of coating flows, computational fluid dynamics applications, optimization methods, workflow execution environments and value-driven design.

Anne Monceaux is a senior research fellow at EADS Innovation Works. She obtained a $\mathrm{PhD}$ in Formal and Theoretical Linguistics from the University Paris 7 in 1993, France and performed lecturing and research at Gaspard Monge Electronic and Computer Science Institute, France until 1997. In 1998 she joined the Simulation, IT \& System Engineering research team at EADS IW. Her research interests are in the fields of Information Systems and Knowledge Management applications for System Engineering.

Alessandro Bertoni is a Ph.D. student in Product Innovation at the Division of Innovation and Design at Luleå University of Technology in Sweden. With a background on Management Engineering he is focusing his research on studying new methods and tools for the assessment and communication of the value of a product or service in the early stages of the design process. He is mainly working in close collaboration with the aerospace industry in the frame of the EU FP7 CRESCENDO project.

Steve Wiseall is a team leader in Cost Modeling Strategy group in Rolls-Royce plc. He obtained his BSc degree in Physics from Loughborough University in 1979 and an EngPhil degree in Laser Systems Design from the same university in 1982. After completing his PhD thesis on Laser Material Interactions, he joined Rolls-Royce plc in 1984. During his tenure in Rolls-Royce plc, he completed a company sponsored MBA degree in 1993 and joined the Design Methods Team in 1995. His research interests are in the fields of optimization, design to manufacture, cost modeling and value-driven design.

Xinwei Zhang is a lecturer at School of Management, Northwestern Polytechnical University, Xi'an, China. He obtained his $\mathrm{PhD}$ degree in Industrial Engineering from LAAS-CNRS laboratory and INSA Toulouse, France in 2012. His research area is about requirements engineering for complex systems and value-driven design. 